\title{
Les analyses
}

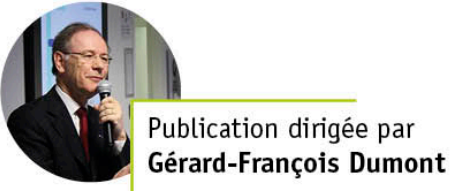

https://www.population-et-avenir.com/les-analyses-de-populationavenir/

\section{Roland Pressat, un démographe éminent de l'équipe d'Alfred Sauvy}

\author{
par \\ Jean-Paul Sardon \\ Directeur de recherche honoraire à \\ l'Institut national d'études démographiques (INED)

\section{Chantal Blayo} \\ Professeure honoraire à \\ l'Université de Bordeaux
}




\section{Roland Pressat, un démographe éminent de l'équipe d'Alfred Sauvy \\ Jean-Paul Sardon et Chantal Blayo}

\section{Résumé}

Roland Pressat (1923-2020) est un éminent représentant des chercheurs forgés dans «l'esprit Sauvy». Sens de la rigueur, qualités de plume et goût pour la vulgarisation. Sa contribution à la diffusion de l'analyse démographique fut considérable et de nombreux démographes, à travers tous les continents, lui doivent leur formation, tant par son enseignement, à Paris et à Montréal, que par ses ouvrages. Son innovation pédagogique fut la création de «devoirs » comme apprentissage à la recherche. Homme de passion, il était également collectionneur de gravures, amateur de vins et de cigares.

\section{Mots-clés}

Pressat; Sauvy; Henry; INED ; IDUP ; Université de Montréal ; enseignement ; analyse démographique ; pratique de la démographie.

\section{Abstract}

Roland Pressat (1923-2020) is a prominent représentative of researchers forged in the "Sauvy spirit ». Sense of rigor, qualities of pen and taste for popularization. His contribution to the dissémination of demographic analysis was considérable and many demographers across all continents, owe him their training, both by his teaching, in Paris and Montreal, and his books. His pedagogical innovation was the création of « homework» as a learning to research. A man of passion, he was also a collector of engravings, a lover of wines and cigars.

\section{Keywords}

Pressat; Sauvy; Henry; INED; IDUP; University of Montreal ; teaching ; demographic analysis; workbook in demography.

\section{Pour citer cette publication :}

\section{To cite this version :}

Sardon, Jean-Paul, Blayo, Chantal, «Roland Pressat, un démographe éminent de l'équipe d'Alfred Sauvy", Les analyses de Population \& Avenir, mars 2020, p. 1-17. [ISSN 2552-2078] 


\section{Roland Pressat, un démographe éminent de l'équipe d'Alfred Sauvy}

Jean-Paul Sardon et Chantal Blayo

\section{Sommaire}

ENFANCE ET FORMATION

ROLAND PRESSAT A L'INED : UN ESSAI TRANSFORME POUR PARTICIPER A

« L'ESPRIT SAUVY »

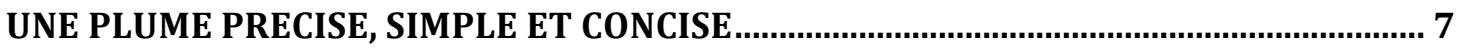

ROLAND PRESSAT ET LE JUSTE USAGE DES MATHEMATIQUES ................................... 8

DES QUALITES AU SERVICE DE L'ENSEIGNEMENT ET DE LA REDACTION DE MANUELS

UNE REMARQUABLE INNOVATION PEDAGOGIQUE

AUCUNE CONCESSION A L'ABSENCE DE RIGUEUR ........................................................14

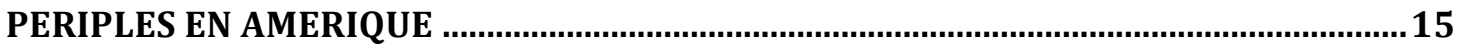

HOMME DE PASSION 
Dès l'origine de l'Institut national d'études démographiques (INED) en 1945, Alfred Sauvy ${ }^{1}$ s'était donné pour but d'éclairer les questions de population par une large diversité de disciplines scientifiques. C'est ainsi qu'il s'entoura de personnalités, au parcours varié, issues pour une grande part de chercheurs déjà présents dans la fondation Carrel$^{2}$, ainsi que de militaires rendus libres par la fin de la guerre.

Cet éclectisme dans le recrutement et l'ampleur de la tâche à accomplir ne pouvaient se réaliser que dans un climat particulier, fait de liberté et d'autonomie, ce qu'il est maintenant convenu d'appeler «l'esprit Sauvy ».

Roland Pressat ${ }^{3}$, dans le numéro de la revue Population consacré aux "Cinquante années de Population», décrit cet état d'esprit, né de la rencontre de l'équipe fondatrice et de la personnalité d'Alfred Sauvy, comme une "manière de gouverner et de mettre les chercheurs dans les conditions les plus propices à leur recherche ». Ainsi écrit-il, dès le début de son article "Entrer à l'INED en 1953 c'était s'intégrer à une équipe, sans rival à cette époque, qui portait la démographie au plus haut niveau ».

Cela se caractérisait par l'encouragement de «la plus large ouverture sur tous les problèmes de population et en laissant, à ses collaborateurs, la plus totale liberté dans la conduite de leurs recherches ».

C'est ce que mit en pratique Roland Pressat tout au long de sa carrière.

${ }^{1}$ Alfred Sauvy (1898-1990), fondateur et premier directeur de l'Ined (1945-1962).

2 Fondation française pour l'étude des problèmes humains, créée sous le gouvernement de Vichy à l'instigation du docteur Alexis Carrel.

${ }^{3}$ Pressat, Roland, « L'INED à ses débuts : L'esprit Sauvy », Population, 1995, 50-6, pp. 1349-1354. 


\section{Enfance et formation}

Roland Pressat est né le 28 juin 1923 dans ce quartier du nord-ouest de Paris qu'est Montmartre d'un père majordome et d'une mère couturière. La famille ayant déménagé à Donzy (Nièvre), il suit des études au collège de Cosne-sur-Loire, puis intègre l'école normale d'instituteurs ${ }^{4}$ du département en répondant ainsi au souhait de ses parents. Le régime de Vichy ayant décidé, en octobre 1941, la fermeture des écoles normales d'instituteurs, il fut contraint d'intégrer, comme boursier, le lycée. Comme Roland Pressat le disait lui-même, ce fut sa chance, car il sentait qu'il avait des capacités en sciences.

C'est ainsi qu'après son baccalauréat, il passa une licence de mathématiques.

Pendant sa période d'études à Paris, il avait été recueilli par Régis Blachère ${ }^{5}$, éminent islamologue, car sa famille était de la Nièvre. Il voua toute sa vie une grande admiration à cet homme d'une grande rigueur morale qu'il définissait comme son bienfaiteur. Son souvenir en fut ravivé, quand il fut victime de problèmes de vision, par la crainte de devenir aveugle comme lui.

Pendant cette période de formation, où il vécut dans un extrême dénuement, Roland Pressat avait obtenu, en 1945, un poste de démonstrateur vacataire au Palais de la découverte et c'est avec un grand enthousiasme qu'Il évoquait cette expérience.

\footnotetext{
${ }^{4}$ Ecole chargée de former les enseignants des écoles maternelles et primaires, remplacées par les Instituts universitaires de formation des maîtres (IUFM en 1990), lorsque la dénomination professeurs des écoles remplace celle d'instituteur, devenues Écoles supérieures du professorat et de l'éducation en 2013.

${ }^{5}$ Régis Blachère (1990-1973), alors professeur d'arabe à l'École nationale des langues orientales (Langues O) de 1935 à 1950.
} 


\section{Roland Pressat à I'INED: un essai transformé pour participer à "l'esprit Sauvy $\Perp$}

Après avoir entamé, après guerre, une carrière de professeur de mathématiques à Paris, comme adjoint d'enseignement, Roland Pressat a souhaité réorienter sa carrière vers la recherche démographique, suite à la lecture d'une petite affiche sur l'INED vue sur un mur du Grand Palais. Dans ce dessein, comme le relate Alain Girard ${ }^{6}$ dans son livre consacré à l'histoire de l'Ined7, en 1953, "C'est lui qui écrit au directeur pour lui demander s'il pourrait l'accueillir ».

Roland Pressat racontait lui-même qu'il avait joint à sa lettre une feuille récapitulant ses diplômes sur la partie gauche et ses emplois à droite, cette présentation synthétique plut à Alfred Sauvy qui accepta de le recevoir et, comme l'écrit Alain Girard ${ }^{8}$, "Sauvy ne lui cache pas qu'il ne l'engage qu'à l'essai pour un an », et lui demande de s'inscrire, en même temps, à l'Institut de statistique de l'université de Paris (ISUP) ${ }^{9}$. Au cours de ce cursus, son premier maître en démographie fut alors Paul Vincent ${ }^{10}$ et il y acquit les premiers éléments du calcul des indicateurs de base, déjà présentés par Michel Huber ${ }^{11}$, son prédécesseur sur ces thématiques ${ }^{12}$.

Il est vrai qu'à l'époque la démographie n'était guère enseignée. Alfred Sauvy, avec son sens de la formule, définissait la démographie comme « une discipline sans maîtres et sans élèves " $^{13}$. La plupart de ceux qui firent partie, à l'origine de l'INED, de l'équipe de chercheurs et furent reconnus par la suite comme des maîtres de la recherche démographique, entrèrent à l'INED, avec pour seul « bagage » le diplôme de leur formation

\footnotetext{
${ }^{6}$ Alain Girard (1914-1996), chercheur à l'Ined et professeur à la Sorbonne à la chaire de démographie à compter de 1964

${ }^{7}$ Girard, Alain, L'INED, Histoire et développement, Paris, Editions de l'INED, 1986, p. 114.

${ }^{8}$ Girard, Alain, L'INED, Histoire et développement, op. citat. P. 114.

${ }^{9}$ Dont il fut diplômé en 1954

${ }^{10}$ Paul Vincent (1912-1979), directeur de recherche à l'Ined, a notamment été fin 1945 chef de la section d'études quantitatives et de conjoncture démographique.

${ }^{11}$ Michel Huber (1876-1947) a notamment enseigné à l'Institut de statistique de Paris et a été Viceprésident de l'Institut international de statistique.

${ }^{12}$ Michel Huber, Cours de démographie et de statistique sanitaire, en 6 fascicules, Paris, Hermann \& Cie, 1938-1941.

${ }^{13}$ Formule citée par Roland Pressat, «L'INED à ses débuts : L'esprit Sauvy », Population, 1995, 50-6, p. 1350 . 
initiale, sans aucune formation particulière dans leur nouveau domaine d'études.

Mais au fur et à mesure du développement de l'administration de la recherche, la mise en place de règles contraignantes pour tout, y compris pour le recrutement des chercheurs, sonna la fin de cet âge d'or de «l'esprit Sauvy ». Les pionniers de cette époque ne furent pas épargnés et, périodiquement, il leur était conseillé de passer une thèse pour renforcer leur position. Ainsi Roland Pressat rapportait-il malicieusement la réponse, faussement, ingénue que Louis Henry fit un jour à un de ces personnages qui lui conseillait de passer une thèse : "Et je serai dans le jury?»

Comme le souligne Gérard Calot: "La foi, celle qui animait Roland Pressat, et dont on dit qu'elle déplace les montagnes, remplaçait avantageusement tous les diplômes imaginables! Et on est pris soudain d'amertume à constater que des candidats à l'entrée à l'INED, comme l'ont été Paul Vincent, Jean Bourgeois-Pichat ${ }^{14}$, Louis Henry ${ }^{15}$ ou Roland Pressat, seraient aujourd'hui par principe refusés pour des raisons probablement justifiables mais ô combien injustifiées et regrettables ! ${ }^{16}$.

\section{Une plume précise, simple et concise}

Arrivé au moment où Jean Bourgeois-Pichat part aux Nations Unies à New York, Roland Pressat seconda Louis Henry dans la préparation et la rédaction de la "situation démographique», tâche dont il s'acquitta pendant plus de dix ans, et qui se poursuivit par l'écriture du Rapport annuel sur la situation démographique de la France ${ }^{17}$.

Dans l'écriture de ses articles, il se distinguait, dans le droit fil de ce qu'exigeait Alfred Sauvy, par la précision, la simplicité et la concision de sa

\footnotetext{
14 Jean Bourgeois-Pichat (1912-1990), notamment directeur de l'Ined de 1962 à 1972.

15 Louis Henry (1911-1991), considéré par Alfred Sauvy comme le créateur de la démographie historique.

${ }^{16}$ Calot, Gérard, «Préface », Population, n6, novembre-décembre 1991. Numéro spécial : Hommage à Roland Pressat : méthodes et applications de l'analyse démographique, p.1363

${ }^{17}$ Le décret $n^{\circ} 73-222$ du 22 février 1973, définissant le statut de l'INED, qui s'était substitué à l'ordonnance de 1945 créant l'INED, avait imposé à l'Institut la rédaction annuel d'un rapport au Parlement sur la situation démographique de la France.
} 
plume, qualités soulignées par Gérard Calot ${ }^{18}$, dans la préface du numéro de Population qui lui est consacré : "Auteur anonyme depuis près de vingt ans $d u$ Rapport annuel au Parlement sur la situation démographique de la France, Roland Pressat a témoigné du souci qui l'animait de présenter les résultats de la recherche sous la forme qui permet d'en élargir la diffusion au plus grand nombre ${ }^{19}$.

\section{Roland Pressat et le juste usage des mathématiques}

Ces qualités d'écriture éclatent dans la rédaction des définitions de son dictionnaire démographique ${ }^{20}$, comme dans tous ses autres travaux. L'une des caractéristiques de ses publications scientifiques, qu'il partageait avec Louis Henry, c'est de ne jamais avoir recours à un bagage mathématique compliqué. Il se plaisait à dire que la recherche démographique ne nécessitait pas plus que la maîtrise des quatre opérations de base.

Cet effort de ne pas céder à la facilité, et à la prétention, de l'utilisation des formules mathématiques et de la notation continue, il le faisait pour des raisons de lisibilité et de clarté, à destination d'un lectorat plus large que celui du seul spécialiste.

Et pourtant il avait cette compétence mathématique, comme il le prouva, après son départ à la retraite, par la rédaction d'un très accessible manuel de démographie mathématique, qu'il donna à publier à l'Association internationale des démographes de langue française ${ }^{21}$.

\footnotetext{
${ }^{18}$ Alors directeur de l'INED.

${ }_{19}$ Calot, Gérard, « Préface », Population, n 6, novembre-décembre 1991, Op. citat., p. 1364.

${ }^{20}$ Pressat, Roland, Dictionnaire de Démographie, Paris, PUF, 1979, 297 p., tabl., annexe.

${ }^{21}$ Pressat, Roland, Eléments de démographie mathématique, AIDELF, 1995, 280 p.
} 
La couverture du dictionnaire de démographie de Roland Pressat (1979)

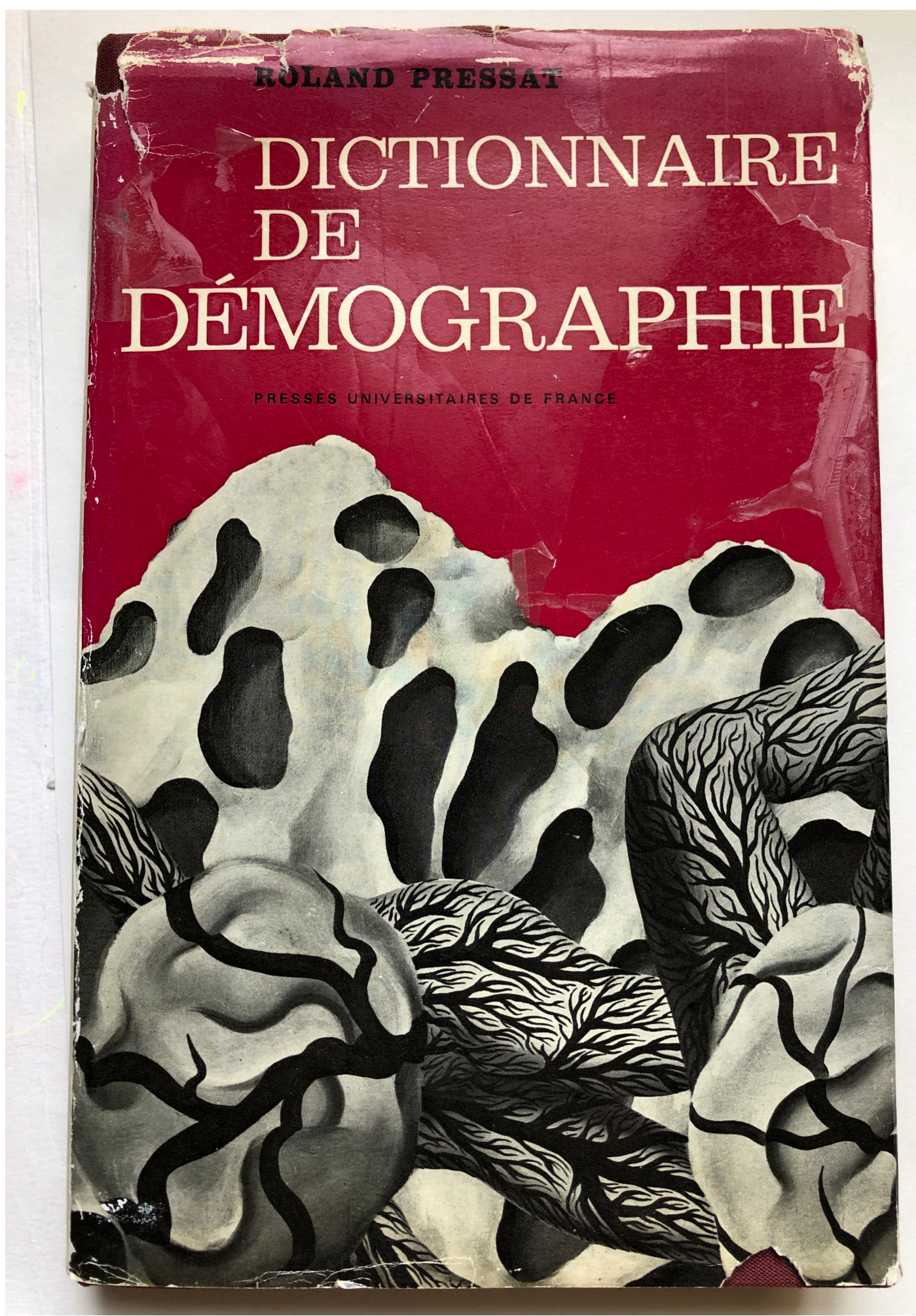


Pour Roland Pressat, les mathématiques étaient avant tout un outil qui devait pouvoir être clairement exprimé également par le langage courant. C'est pourquoi, il était fasciné par la qualité littéraire des manuels de mathématiques rédigés par les grands mathématiciens du XIX ${ }^{\mathrm{e}}$ et du début du XXe siècle, et qu'il achetait régulièrement.

C'est sans doute pour les mêmes raisons qu'il se lia d'amitié avec Marc Barbut ${ }^{22}$, qu'il invita à donner des cours à l'INED au milieu des années 1960. Il échangeait régulièrement avec lui sur des problèmes statistiques, pour vérifier auprès de lui la justesse de ses raisonnements critiques à l'égard de certaines publications. Ces travaux débouchèrent, en 2001, sur la publication de deux articles dans sa revue Mathématiques et sciences humaines ${ }^{23}$.

Il entretint également des relations longues et régulières avec Nathan Keyfitz $^{24}$, autre grande figure de la démographie mondiale, qui lui aussi disparut dans sa 97ème année, en 2010.

Démographe de l'équipe d'Alfred Sauvy, il le fut, à la fois, par la qualité de sa plume que nous venons d'évoquer, par la diversité de ses centres d'intérêt, dont un coup d'œil à sa bibliographie ${ }^{25}$ suffit à rendre compte de l'extrême richesse ${ }^{26}$, mais également par son goût pour la vulgarisation.

Il contribua ainsi à de nombreuses publications, dont la revue Population et Avenir, mais surtout au Concours médical dans lequel il publia près de 110 articles en l'espace de 38 années.

\footnotetext{
${ }^{22}$ Marc Barbut (1928-2011), universitaire.

${ }^{23}$ Pressat, Roland, "Christian Huygens et la table de mortalité de Graunt », Mathématiques et sciences humaines, 153, 2001, pp. 29-36 ; Pressat, Roland, «Quelques vues de Leibnitz en relation avec la durée de la vie humaine ", Mathématiques et sciences humaines ,155-156, 2001, pp. 43-51.

${ }^{24}$ Nathan Keyfitz (1913-2010), universitaire canadien dont la carrière, poursuivie aux Etats-Unis, le conduisait à enseigner à Harvard.

${ }^{25}$ https://www.ined.fr/fichier/s_rubrique/29903/biblio.r.pressat.fr.pdf

$26 \frac{1}{11}$ fut l'un des premiers démographes occidentaux à s'intéresser à l'URSS et à nouer des relations avec les démographes russes.
} 


\section{Des qualités au l'enseignement et de la rédaction de manuels}

Dans diverses universités existait, dans la seconde moitié des années 1940, un enseignement consacré aux problèmes de population, sous l'aspect propre à la discipline dans laquelle cet enseignement s'insérait. Les universitaires, qui nouaient des liens assez étroits avec l'INED qui leur fournissait un vivier de conférenciers, ressentirent la nécessité de se rassembler dans une « Réunion des directeurs d'instituts universitaires et centres interfacultés de démographie », structure informelle créée en 1948 et présidée alors par le sociologue Jean Stœtzel ${ }^{27}$.

Après une intense mobilisation de cette « Réunion », et du géographe Pierre George notamment ${ }^{28}$, et après la création de l'Institut d'études démographiques de l'Université de Bordeaux (IEDUB) dirigée par Jean Stœtzel, fut créé en 1957 l'Institut de démographie de l'Université de Paris (IDUP), dont la direction fut confiée à Alfred Sauvy, alors directeur de l'INED. L'IEDUB et l'IDUP sont alors les deux instituts habilités à délivrer des diplômes de 3 ème cycle, le diplôme de Démographie générale et le diplôme d'Expert démographe. A Paris, l'enseignement de l'analyse démographique revint à Louis Henry qui confia alors les travaux pratiques à Roland Pressat. Ce dernier est alors également chargé, par Alfred Sauvy, de rédiger un manuel de démographie à l'usage des étudiants ${ }^{29}$.

Ce livre connaîtra dès sa parution un succès immédiat, tant en France que dans le monde, comme l'atteste le nombre important de traductions, pas toujours en accord avec l'auteur ou l'éditeur.

Sans conteste, les qualités pédagogiques de son auteur et la qualité de son écriture furent les moteurs incontestables de cette diffusion.

\footnotetext{
27 Jean Stœtzel (1910-1987), notamment professeur de psychologie sociale à la Sorbonne (1955-1978), introducteur en France de la méthode des sondages d'opinion.

${ }^{28}$ Qui rédigea, au nom de la Réunion des directeurs, une proposition de création d'un enseignement supérieur de $3^{\text {ème }}$ cycle de démographie. Cf. Girard, Alain, L'INED, Histoire et développement, Pa ris, Editions de l'INED, 1986, pp. 208-209.

${ }^{29}$ Pressat, Roland, L'analyse démographique. Méthodes, résultats, applications, Paris, PUF, 1961, 403 
À compter de 1965, Roland Pressat partage avec Louis Henry le cours d'analyse démographique. Celui-ci fera l'objet d'une publication ${ }^{30}$.

En 1969, Roland Pressat refonde ${ }^{31}$ totalement son premier manuel, essentiellement inventaire des principaux indicateurs, en y intégrant son cours de «Principes » inspirés des travaux de Louis Henry publiés dans Population.

La contribution de Roland Pressat à la diffusion de cette discipline qu'est la démographie fut considérable par son sens pédagogique. Non seulement, il l'enseigna de nombreuses années à l'Institut de démographie de l'Université de Paris, ainsi qu'à Montréal, mais il rédigea également de nombreux ouvrages.

Ainsi de nombreux démographes, à travers tous les continents, lui doivent-ils leur formation, soit directement par son enseignement, soit à travers ses ouvrages.

\section{Une remarquable innovation pédagogique}

Dans ce domaine, il avait un véritable talent pédagogique qu'il expliquait modestement en disant que, n'étant pas un génie, il pouvait avoir des difficultés pour comprendre tel ou tel raisonnement, et, de ce fait, était plus à même de les expliquer car il savait où se situaient les obstacles que rencontraient les étudiants.

Mais sa contribution majeure à l'enseignement de la démographie fut la création de «devoirs ». Ces «devoirs» n'étaient pas des exercices d'applications des indicateurs démographiques, mais des mini-recherches guidées par des réponses à des questions au fil desquelles des problèmes concrets trouvaient leur solution. Aussi, peut-on dire que son chefd'œuvre dans le domaine de l'enseignement de la démographie est incontestablement sa "Pratique de la démographie.», recueil de ces devoirs, parue en 1967, «qui fondera sa réputation ${ }^{32}$. Cette innovation,

\footnotetext{
${ }^{30}$ Pressat, Roland, Principes d'analyse. Cours d'analyse démographique de l'IDUP, Paris, INED, 1966, $155 \mathrm{p}$.

${ }^{31}$ Pressat, Roland, L'analyse démographique, concepts, méthodes, résultats, Paris, PUF, 1969, 323 p.

32 Girard, Alain, L'INED, Histoire et développement, Paris, Editions de I'INED, 1986, pp. 210.
} 
véritable révolution dans la manière d'enseigner l'analyse démographique, non plus avec un catalogue de recettes, mais comme un apprentissage à la recherche où l'on avance pas à pas dans la réflexion, n'a toujours pas la diffusion qu'elle mérite.

Les disciples de Roland Pressat, comme Yves Péron ${ }^{33}$ ou Chantal Blayo, et quelques-uns de leurs élèves, continuèrent dans cette voie avec la création d'autres devoirs. Les principaux démographes analystes, encore en activité ou non, ont tous été formés de cette façon, mais l'exigence des nouveaux diplômes rend de plus en plus difficile ce type d'enseignement réellement innovant et formateur qui exige que l'enseignement de l'Analyse démographique ne soit pas noyé dans de vastes formations en Sciences humaines.

Les talents de Roland Pressat dans l'enseignement de la démographie, et la renommée importante que ses ouvrages procurèrent, conduisirent les organisateurs des conférences ou congrès internationaux de démographie à lui confier souvent la présidence des séances consacrées à l'enseignement de la démographie ${ }^{34}$.

À partir de 1973, Roland Pressat partagea son enseignement de la démographie entre Paris et Montréal où il fut professeur invité jusqu'à sa nomination, en 1974, comme professeur titulaire au Département de démographie de l'Université de Montréal, poste qu'il occupa jusqu'en 1978.

$\mathrm{Au}$ cours de son séjour canadien, Roland Pressat forma nombre de démographes québécois, y amplifiant encore la diffusion de ce que l'on a coutume d'appeler "l'école française de démographie », qui y était déjà bien présente car nombre des enseignants étaient passés par l'IDUP et/ou l'INED.

Roland Pressat mit également à profit ce séjour québécois pour rédiger son Dictionnaire de démographie et dans son Avant-propos, il souligne, en conclusion, l'importance de celui-ci dans l'écriture de son livre : "Il nous est agréable de conclure en reconnaissant tout ce que nous

\footnotetext{
${ }^{33}$ Professeur honoraire à l'Université de Montréal.

${ }^{34}$ Cf. Girard, Alain, L'INED, Histoire et développement, Paris, Editions de I'INED, 1986, pp. 214 ; Pressat, Roland, Avancement de l'enseignement et de la recherche démographique en Europe. Conseil de l'Europe, $2^{\text {ème }}$ conférence démographique européenne, Strasbourg, 31 août-7 sept. 1971, 207 p. 
devons, dans l'élaboration de cet ouvrage, aux conditions de travail qui nous ont été offertes au département de démographie de l'Université de Montréal » ${ }^{35}$ en précisant en fin le lieu et la date: "Montréal, le 14 septembre $1978 »$.

Ce dictionnaire reflète le désir de simplicité, de précision et de rigueur qui caractérisait son écriture.

\section{Aucune concession à l'absence de rigueur}

Ce sens de la rigueur, auquel il n'acceptait aucune concession, le faisait bondir quand il lisait des publications démographiques avec des fautes d'analyse ou qui n'utilisaient pas les termes démographiques de manière appropriée ou qui utilisaient des mots du langage courant en lieu et place des termes consacrés.

Ce sens critique, son besoin de rigueur, il les a gardés tout au long de sa vie. En 2018, à l'âge de 95 ans, Roland Pressat mobilise toute son énergie pour mener à son terme l'écriture de son dernier ouvrage, Observations sur le Dictionnaire de démographie et des sciences de la Population ${ }^{36}$, critique d'un dictionnaire démographique ${ }^{37}$ qui ne faisait pas toujours preuve de la rigueur nécessaire.

Conscient que cet ouvrage, dans sa version papier, ne pouvait avoir qu'une diffusion limitée, Roland Pressat accepte avec plaisir la proposition de publication numérique faite par Gérard-François Dumont.

Ainsi le dernier ouvrage de Roland Pressat a paru sous forme numérique dans la publication Les analyses de Population \& Avenir ${ }^{38}$.

\footnotetext{
${ }^{35}$ Pressat, Roland, Dictionnaire de démographie, Paris, PUF, 1979, 297 p.

${ }^{36}$ Edité en 2018 à compte d'auteur et diffusé également dans Les analyses de Population \& Avenir, janvier 2019, 70 pages, ISSN 2552- 2078 (https://www.population-et-avenir.com/les-analyses-de-populationavenir/)

Meslé, France, Toulemon, Laurent, Véron, Jacques (direction), Dictionnaire de démographie et des sciences de la Population, Paris, Armand Colin, 2011, 528 p.

${ }^{38}$ https://www.population-et-avenir.com/les-analyses-de-population-avenir/
} 


\section{Périples en Amérique}

C'est peut-être son séjour au Canada qui donna à Roland Pressat le goût des grands espaces et des grands voyages. Aussi parcourut-il plusieurs fois, avec son épouse Cécile, les États-Unis au volant de voitures de location. C'est ainsi qu'il suivit la mythique Route 66, de Chicago à Los Angeles.

Roland Pressat adorait conduire aux États-Unis, où il louait la tranquillité et la gentillesse des conducteurs américains qui contrastaient avec l'énervement des chauffeurs français et parisiens.

Il visita également deux fois Cuba, avec son épouse et ses petitsenfants et, au cours de l'une de ses pérégrinations, voulant désaltérer sa famille, dans un petit village, il entra dans un bar dans lequel Fidel Castro mangeait avec quelques amis. Les petits-enfants ne purent s'empêcher, faisant fi de l'étiquette, de prendre une photo du « Lider Maximo ».

\section{Homme de passion}

Par-delà son parcours démographique et académique, Roland Pressat était avant tout un homme de passion (comme de rigueur, avec le sens du partage).

Passion tout d'abord pour la Démographie, en particulier pour l'Analyse démographique dans laquelle sa rigueur trouvait tout son épanouissement.

La rigueur, c'est sans doute ce qui l'a séduit dans une autre de ses passions, la Gravure, et encore plus précisément dans l'œuvre cubiste de Jacques Villon ${ }^{39}$, passion qu'il partageait avec son épouse Cécile. Et ne pouvant faire les choses qu'intensément, il en est devenu l'un des grands collectionneurs et l'un des spécialistes. C'est ainsi qu'il rédigea, en 1989, la plaquette d'une exposition qui lui était consacrée ${ }^{40}$.

\footnotetext{
39 Jacques Villon (1875-1963).

${ }^{40}$ Roland Pressat, « Jacques Villon. L'œuvre gravé. Exposition autour d'une collection », Paris, 1989.
} 
Mais cette passion l'a amené à grandement élargir sa collection, des grands maîtres anciens jusqu'à des graveurs contemporains, et ce jusqu'à une date récente. Il s'intéressa également à la tapisserie et plus particulièrement à celles de Lurçat.

Son sens du partage l'amenait à organiser régulièrement des visites de ses nouvelles acquisitions ou de ses pièces préférées, au cours desquelles il était intarissable sur l'histoire particulière de chacune des acquisitions qu'il avait faites, sur la composition de la gravure, sa technique, les circonstances de sa création ou les différentes collections auxquelles la pièce avait appartenu.

Parmi ses lieux d'exposition préférés, l'Institut néerlandais, devenu Fondation Custodia, dans le $7^{\mathrm{e}}$ arrondissement de Paris, occupait la première place. Il vouait une grande admiration à son fondateur Frits Lugt, amateur d'art et collectionneur avisé. Cette admiration l'avait amené à se lancer, pour sa seule satisfaction personnelle, dans la traduction française de sa biographie, de 473 pages, parue en Anglais en 2012.

De même, en littérature, il aimait faire partager ses goûts, en particulier son admiration pour Paul Léautaud ${ }^{41}$, et son Journal littéraire, dont il appréciait la verve, la spontanéité, le franc-parler et la causticité.

Roland Pressat était également un grand amateur de vins de Bordeaux, qu'il se plaisait là aussi à expliquer et à partager avec ses amis. Pendant de nombreuses années, il fit partie d'un groupe de familiers invités à la table de Jean-Pierre Mouiex, propriétaire de Pétrus et amateur d'art, qui lui permit ainsi de voir l'une des versions de la petite danseuse de Degas.

Si son inclinaison naturelle l'entraînait vers le Bordelais, il parlait aussi avec enthousiasme des qualités des vieux Armagnac, vieux Madère et autres vieux Rhum.

La dernière en date de ses passions fut le cigare pour lequel son envie de partager ce plaisir avec d'autres passionnés, se traduisit par son adhésion au Bordeaux Havane Club, hébergé au Bistrot du Sommelier, créé

\footnotetext{
${ }^{41}$ Paul Léautaud (1972-1956).
} 
par Hervé Valverde ${ }^{42}$, où il se rendait chaque mois. Le dimanche, en famille, il sacrifiait au rite de fumer son cigare après le repas, comme souvent avec ses invités, toujours désireux de faire de nouveaux adeptes, car « un cigare ne se fume pas tout seul, c'est un plaisir qui se partage ».

Tout ce qu'il entreprenait, Roland Pressat le faisait intensément, cherchant à tout savoir pour mieux embrasser le domaine, rencontrant des personnages divers liés à ses centres d'intérêt, collectionneurs, graveurs, producteurs de vin, visitant usine de cigares ou manufacture de tapisserie.... Mais il cherchait toujours à partager ses rencontres et à communiquer ses passions, parlant notamment des dernières expositions qu'il avait visitées, de l'Institut Néerlandais qu'il affectionnait particulièrement ou des gravures qu'il avait vues chez un de ses marchands ou dans une salle de vente.

Par sa production de manuels, Roland Pressat reste, des décennies après leur publication, et restera encore pour longtemps, avec Louis Henry, une référence incontournable dans la formation de générations de démographes. Mais par-delà son activité scientifique, par le large spectre de ses centres d'intérêt, et l'intensité de son investissement dans ces derniers, Roland Pressat apparaît véritablement comme un parfait disciple d'Alfred Sauvy.

Jean-Paul Sardon et Chantal Blayo

42 Dont il aurait fait la connaissance dans un vol pour Cuba. 


\section{La publication}

\section{Les analyses de Population \& Avenir}

[ISSN 2552-2078]

a pour objet de diffuser gratuitement des analyses sur des thèmes diversifiés (fécondité, pauvreté, migration, immigration, intégration, politique familiale, logement, logement social, francophonie, géopolitique, frontière, méthodologie...), et sur des territoires variés précisés dans le titre de la publication (Europe, Union européenne, Afrique, Arabie saoudite, Casamance, Chine, Gambie, Sénégal, France, régions françaises, Corse,...) ou étudiés au sein du thème considéré (Brésil, Maroc, Venezuela...).

Toutes Les analyses de Population \& Avenir sur: https://www.population-et-avenir.com/les-analyses-de-populationavenir/

\section{Les analyses de Population \& Avenir}

\section{Liste des publications}

«Roland Pressat, un démographe éminent de l'équipe d'Alfred Sauvy ? ", Les analyses de Population \& Avenir, mars 2020.

«Le nouveau recensement français : un déficit de jeunes enfants!», Les analyses de Population \& Avenir, janvier 2020. 
«Trois scénarios pour l'avenir de l'Union européenne », Les analyses de Population \& Avenir, décembre 2019.

«Les retraites en Europe: quelles perspectives?», Les analyses de Population \& Avenir, décembre 2019.

"Alfred Sauvy's Working Method», Les analyses de Population \& Avenir, juin 2019.

«La frontière Sénégal-Gambie dans le contexte du conflit en Casamance : mobilités, flux transfrontaliers et géopolitique », Les analyses de Population \& Avenir, juin 2019.

«La francophonie, bilan et perspectives à l'aune des rapports Graddol », Les analyses de Population \& Avenir, 2019.

«Les migrations internationales et l'Afrique : des logiques Sud-Nord ou Sud-Sud ? », Les analyses de Population \& Avenir, 2019.

«Observations sur le Dictionnaire de démographie et des sciences de la Population », Les analyses de Population \& Avenir, 2019.

«La reprise de la fécondité au milieu des années 1930, phénomène non perçu des observateurs du temps?", Les analyses de Population \& Avenir, 2019.

«Immigration: la question de l'intégration dans un contexte fondamentalement nouveau », Les analyses de Population \& Avenir, 2017.

«La pauvreté dans le monde: réponses inopérantes et solutions efficientes », Les analyses de Population \& Avenir, 2016.

«China : a sustainable model ?», Les analyses de Population \& Avenir, 2016.

«Chine : un modèle durable? », Les analyses de Population \& Avenir, 2016.

«Régions françaises: des géants géographiques aux attributions minuscules », Les analyses de Population \& Avenir, décembre 2015. 
"Family policies \& Europe's demographic future», Les analyses de Population \& Avenir, 2015.

«Politiques familiales et avenir démographique de l'Europe», Les analyses de Population \& Avenir, 2015.

«La «crise» des migrants, l'opération anti-passeurs «Sophia » de l'UE et l'ONU », Les analyses de Population \& Avenir, 2015.

«Europe: des disparités considérables dans les évolutions démographiques », Les analyses de Population \& Avenir, juillet 2015.

«Le logement et le logement social en France: éléments de diagnostic », Les analyses de Population \& Avenir, juin 2015.

« Territoires : un fonctionnement radial ou réticulaire?», Les analyses de Population \& Avenir, mai 2015.

"General de Gaulle, Alfred Sauvy and the French Institute for population studies (INED) (1945-1962) », », Les analyses de Population \& Avenir, may 2014.

«Le général de Gaulle, Alfred Sauvy et l'Institut national d'études démographiques (1945-1962)», », Les analyses de Population \& Avenir, mai 2014.

«Women and «Human Rights» in Saudi Arabia», Les analyses de Population \& Avenir, septembre 2012.

«Le nouveau recensement de la population de la France et les améliorations nécessaires », Les analyses de Population \& Avenir, 2008.

«Pourquoi la Corse a-t-elle voté «non» au référendum portant sur une collectivité territoriale unique?", Les analyses de Population \& Avenir, 2003. 


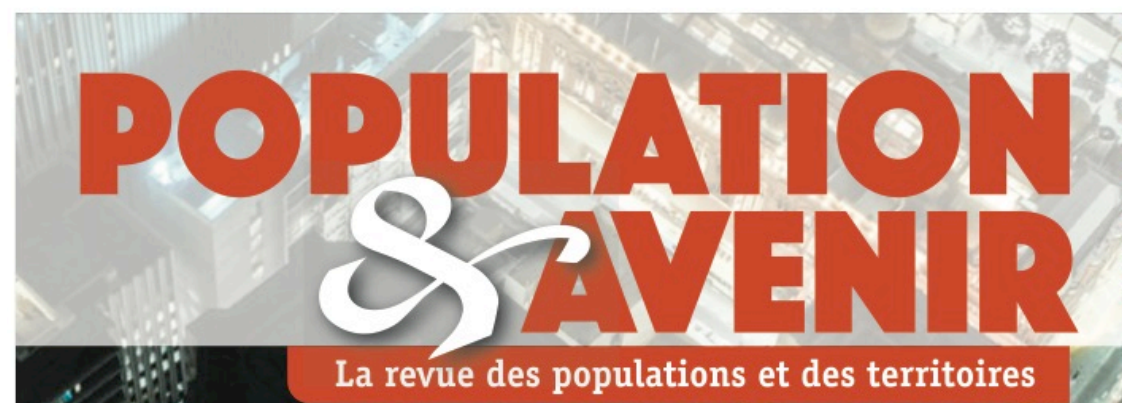

Pour suivre la revue Population \& Avenir :

https://www.population-et-avenir.com/abonnementannuel/abonnement-revue-population-avenir/

Population \& Avenir est la seule revue française et européenne se consacrant exclusivement aux questions géodémographiques et à leurs impacts territoriaux, économiques et sociaux.

Son objet consiste à présenter les évolutions en France, en Europe et dans le monde sous l'éclairage révélateur de la géographie de la population et de la science démographique.

Dans ce dessein, cinq fois par an, Population \& Avenir présente clairement à ses lecteurs le privilège d'informations, d'analyses, de réflexions et d'argumentaires, illustrés par des cartes, des graphiques, des schémas...

En outre, chaque numéro de Population \& Avenir propose un exercice pédagogique, conforme aux programmes de l'enseignement, amplement illustré, et comprenant un corrigé des questions traitées. Cet exercice s'adresse aux professeurs d'histoire-géographie et de sciences économiques et sociales, ainsi qu'aux élèves et aux étudiants devant préparer des examens ou des concours. Il est également fort apprécié de tous les lecteurs qui y voient une sorte de trivial pursuit enrichissant les connaissances tout en stimulant les capacités d'analyse et de synthèse de chacun. 


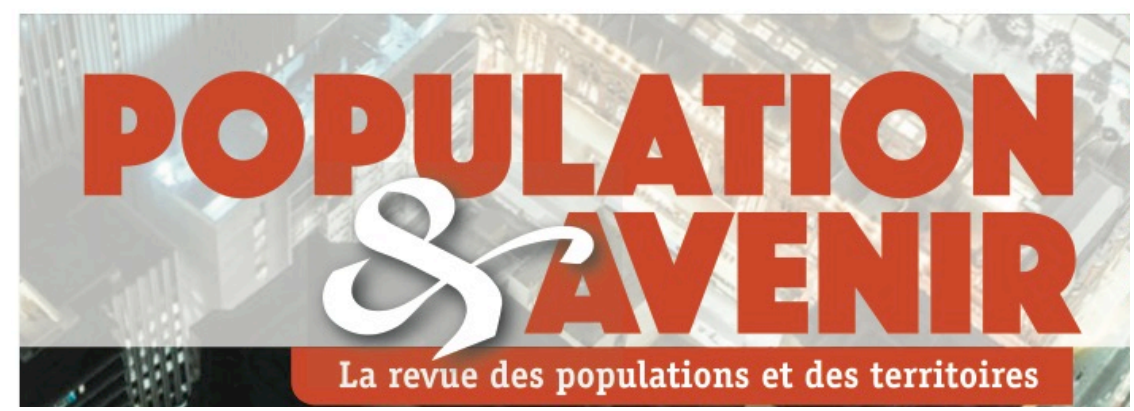

Pour suivre la revue Population \& Avenir:

https://www.population-et-avenir.com/abonnementannuel/abonnement-revue-population-avenir/

La recherche d'un thème ou d'un espace géographique traité dans la revue Population \& Avenir peut s'effectuer sur les sites :

www.population-et-avenir.com

http://population-et-avenir.cairn.info

Une partie des articles et dossiers publiés dans la revue Population \& Avenir peuvent être acquis selon un format numérique sur le site de Cairn info :

https://www.cairn.info/revue-population-et-avenir.htm

Les sommaires de la revue Population \& Avenir en langue anglaise et quelques articles en anglais (english) sont accessibles à l'adresse : https://www.cairn-int.info/journal-population-et-avenir.htm

Pour suivre les publications de l'

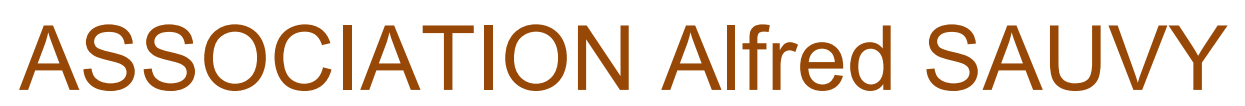

Pour l'étude, la diffusion et la prolongation de l'œuvre d'Alfred Sauvy

Cf. http://www.fourastie-sauvy.org 


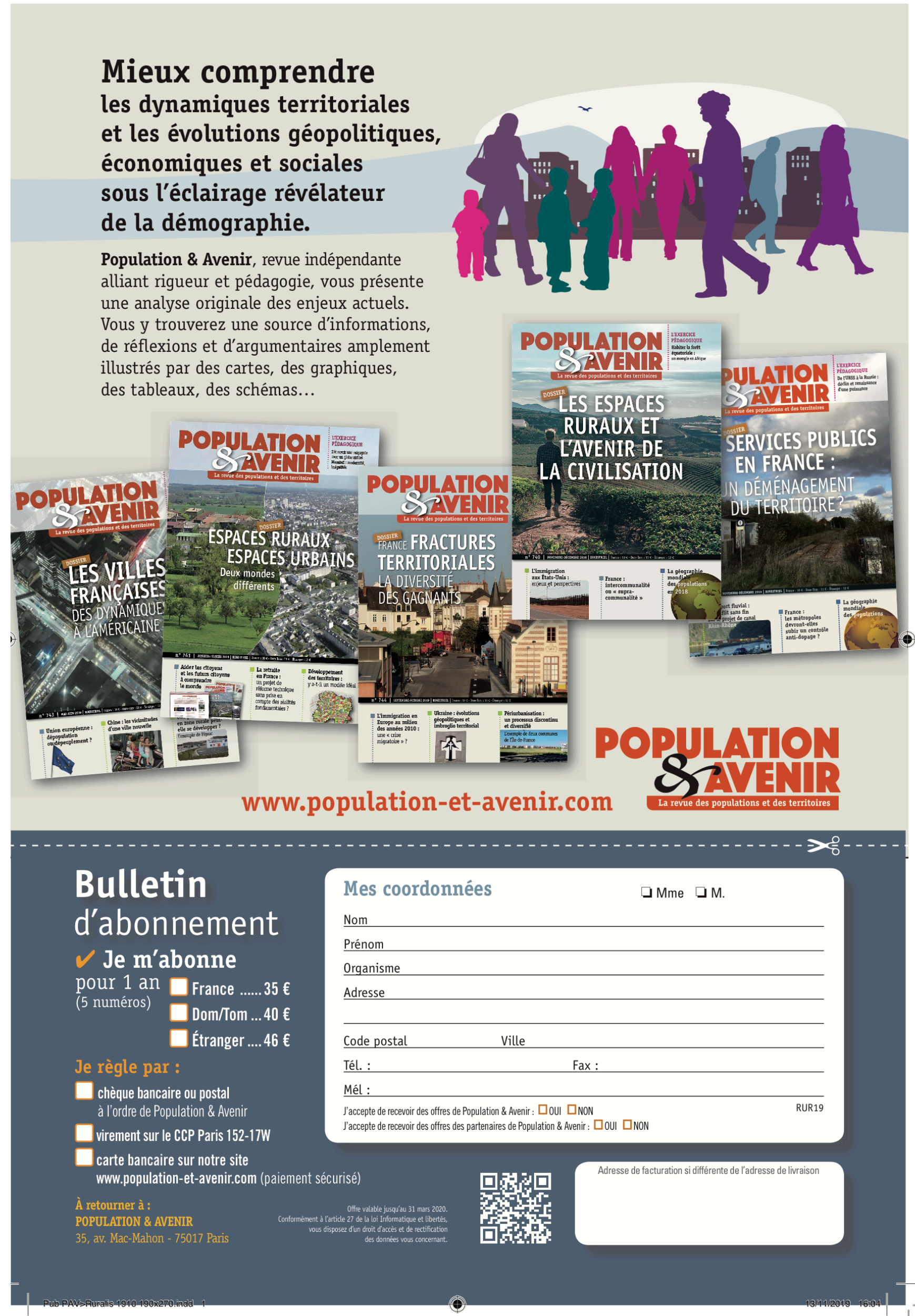

